\title{
Correction to: Evaluation of the presence of Paenibacillus larvae in commercial bee pollen using PCR amplification of the gene for tRNA ${ }^{\text {Cys }}$
}

Vicente Daniel Moreno Andrade ${ }^{1}$. José Luis Hernández Flores ${ }^{2}$ • Miguel Angel Ramos López ${ }^{1}$. Andrés Cruz Hernández ${ }^{3}$. Sergio Romero Gómez ${ }^{1}$. Rosa Paulina Calvillo Medina ${ }^{1}$ - Ana Gabriel Estrada Martínez ${ }^{1}$. Juan Caballero Pérez ${ }^{1}$. Iván Arvizu Hernández ${ }^{1}$. Erika Álvarez Hidalgo ${ }^{1}$. Claudia Álvarado Osuna ${ }^{4}$. George H. Jones ${ }^{5}$. Juan Campos Guillén ${ }^{1}$

Published online: 23 April 2019

(C) Sociedade Brasileira de Microbiologia 2019

Correction to: Brazilian Journal of Microbiology https://doi.org/10.1007/s42770-019-00039-9

In the article mentioned above an author's name was misspelled.

The correct author name reads as follows:

Rosa Paulina Calvillo Medina.

We apologize for the inconvenience.

Publisher's note Springer Nature remains neutral with regard to jurisdictional claims in published maps and institutional affiliations.

The online version of the original article can be found at https://doi.org/ 10.1007/s42770-019-00039-9

Juan Campos Guillén

juan.campos@uaq.mx

1 Facultad de Química, Universidad Autónoma de Querétaro, Cerro de las Campanas S/N, 76010 Santiago de Querétaro, QRO, Mexico

2 Laboratorio de Bioseguridad y Análisis de Riesgo, Departamento de Ingeniería Genética, Centro de Investigación y de Estudios Avanzados del IPN, CP 36824 Irapuato, GTO, Mexico

3 Escuela de Agronomía, Universidad De La Salle Bajío, Campus Campestre, León, GTO, Mexico

4 CIATEJ, Camino Arenero 1227, El Bajio del Arenal, 45019 Zapopan, Jalisco, Mexico

5 Department of Biology, Emory University, Atlanta, GA, USA 